\title{
Thermal Conductivity of Uranium Nitride and Carbide
}

\author{
B. Szpunar' ${ }^{1}$ and J. A. Szpunar ${ }^{2}$ \\ ${ }^{1}$ Department of Physics and Engineering Physics, University of Saskatchewan, Saskatoon, SK, Canada S7N 5E2 \\ ${ }^{2}$ Department of Mechanical Engineering, University of Saskatchewan, Saskatoon, SK, Canada S7N 5A9
}

Correspondence should be addressed to B.Szpunar; b.szpunar@usask.ca

Received 29 May 2014; Revised 25 July 2014; Accepted 31 July 2014; Published 1 September 2014

Academic Editor: Jorge M. Maia

Copyright (C) 2014 B. Szpunar and J. A. Szpunar. This is an open access article distributed under the Creative Commons Attribution License, which permits unrestricted use, distribution, and reproduction in any medium, provided the original work is properly cited.

\begin{abstract}
We investigate the electronic thermal conductivity of alternative fuels like uranium nitride and uranium carbide. We evaluate the electronic contribution to the thermal conductivity, by combining first-principles quantum-mechanical calculations with semiclassical correlations. The electronic structure of UN and UC was calculated using Quantum Espresso code. The spin polarized calculations were performed for a ferromagnetic and antiferromagnetic ordering of magnetic moments on uranium lattice and magnetic moment in UC was lower than in UN due to stronger hybridization between $2 p$ electrons of carbon and $5 f$ electrons of uranium. The nonmagnetic electronic structure calculations were used as an input to BolzTrap code that was used to evaluate the electronic thermal conductivity. It is predicted that the thermal conductivity should increase with the temperature increase, but to get a quantitative agreement with the experiment at higher temperatures the interaction of electrons with phonons (and electron-electron scattering) needs to be included.
\end{abstract}

\section{Introduction}

The recent tragic accident in Fukushima clearly illustrates the risks associated with the present design of reactors based on uranium dioxide $\left(\mathrm{UO}_{2}\right)$ fuel and justifies research towards a safer fuel. Pioro et al. [1] demonstrate that the traditional urania fuel is not suitable for some designs of new generation reactors due to its low thermal conductivity (e.g., the estimated fuel centerline temperature for super critical water reactor (SCWR) surpasses the industry accepted limit of $1850^{\circ} \mathrm{C}(2123 \mathrm{~K})$. Uranium nitride (UN) and uranium carbide (UC) have been proposed as possible safer fuels. UC and UN are advanced types of nuclear fuel since they have not only higher thermal conductivity but also lower linear expansion coefficients and are more compatible with fuel cladding materials [2].

The thermal conductivity of $\mathrm{UN}$ is compared with $\mathrm{UO}_{2}$ in [3]. The researchers show that in contrast to a typical ceramic (e.g., $\mathrm{UO}_{2}$ ) the thermal conductivity of $\mathrm{UN}$ increases with temperature due to a large electronic transport. Additionally, UN melting temperature increases with pressure, which prevents dissociation of nitrogen gas and can be as high as $3035 \mathrm{~K}$ at $1 \mathrm{~atm}[3]$.
UC also has an advantage over $\mathrm{UO}_{2}$ as a fuel for fast reactors due to about 2.6 times higher thermal conductivity $[2,3]$. The melting temperature of UC is slightly lower ( 2623 K [4], 2638 (165) K-2780 (25) K [2]) but still well above $2000 \mathrm{~K}$.

We present here preliminary studies of the thermal conductivity of these advanced types of nuclear fuel.

\section{Electronic Structure}

To evaluate the electronic structure of UN and UC, we use Quantum Espresso (QE) code [5] (see Appendix) as there is already an interface provided between QE and Boltzmann Transport Properties (BoltzTraP) code [6] that will be used here to evaluate electronic transport.

The electronic structure of UC and UN has been studied before (see, e.g., $[7,8]$ ) and researchers note that neither local density approximation (LDA) nor LDA with Hubbard $\mathrm{U}$ corrections is able to adequately describe its electronic structure around Fermi energy that is dominated by strongly correlated $5 \mathrm{f}$ electrons of uranium with antiferromagnetic ordering. However, in spite of the noted deficiencies, many 


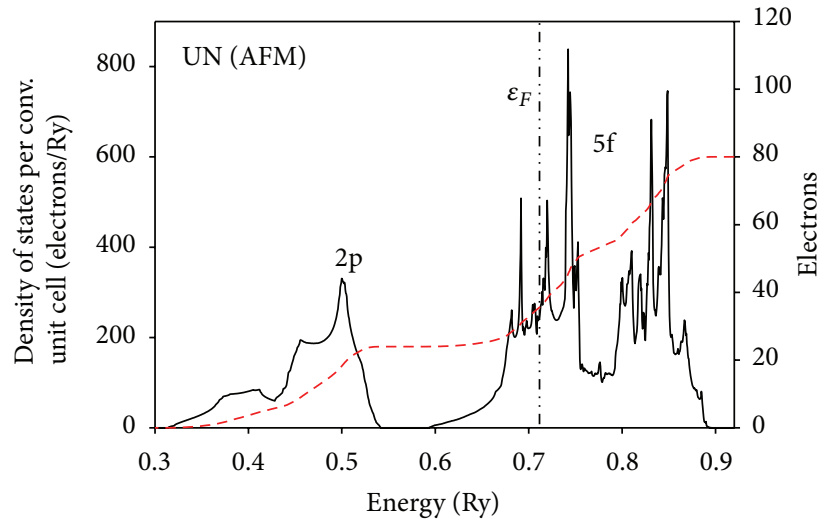

(a)

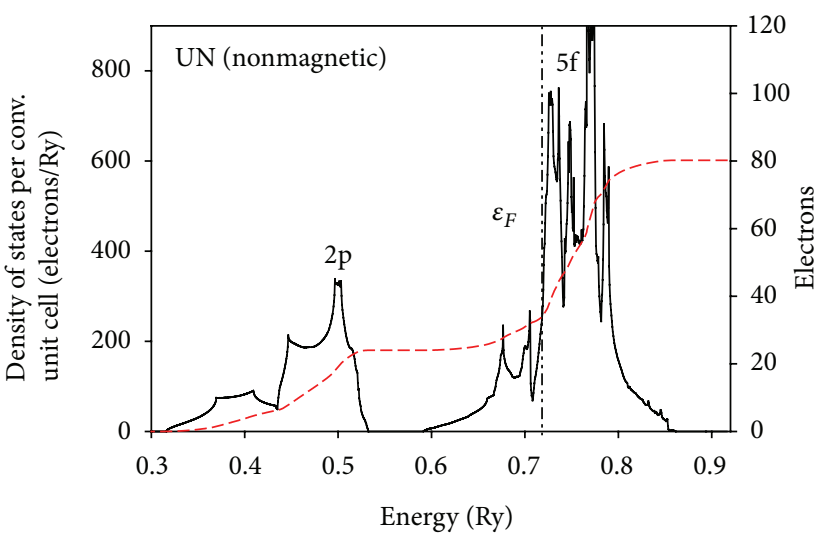

(b)

FIgURE 1: The calculated total (black, solid line) electron density of states of UN using PBE functional for antiferromagnetic (a) and nonmagnetic (b) states and conventional unit cell. The densities of states, integrated over energy, are shown by red dashed lines.

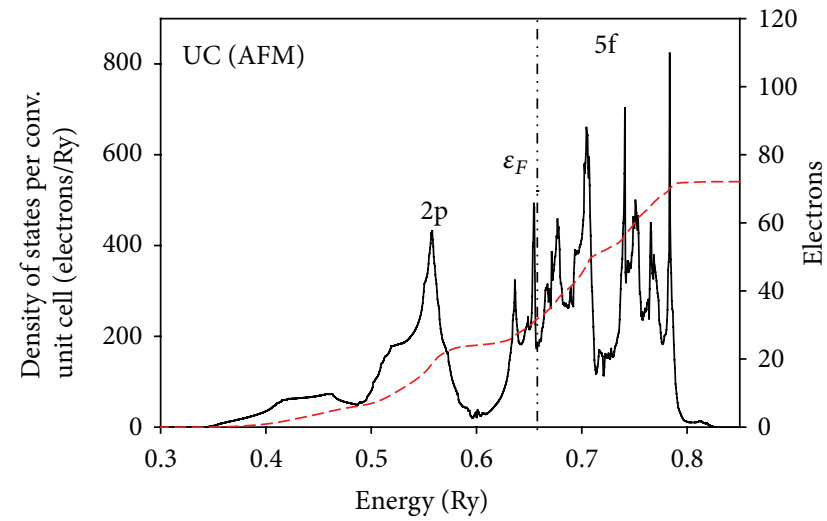

(a)

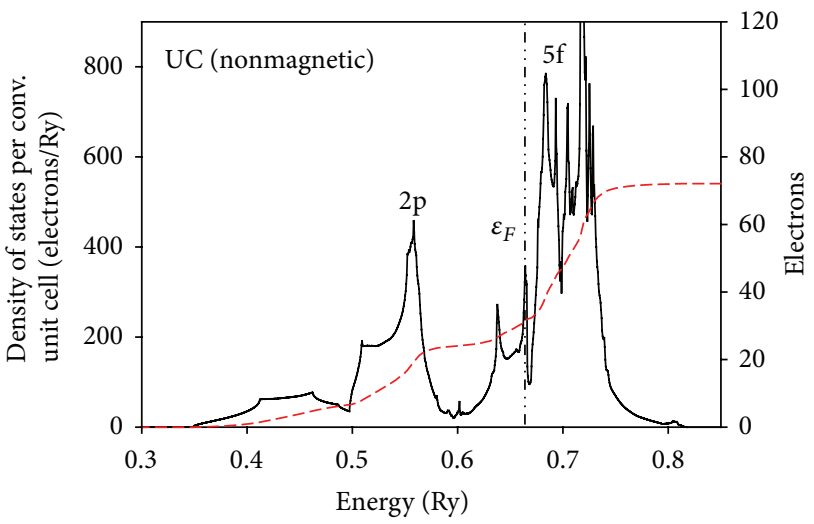

(b)

FIGURE 2: The calculated total (black, solid line) electron density of states of UC using PBE functional for antiferromagnetic (a) and nonmagnetic (b) states and conventional unit cell. The densities of states, integrated over energy, are shown by red dashed lines.

properties can be evaluated in agreement with the experiment, for example, phonon dispersion [8].

2.1. Uranium Nitride. Similar to urania, $\mathrm{UN}$ has a cubic structure $(F m \overline{3} m$ symmetry). At low temperatures (below Néel temperature $T_{N}=53 \mathrm{~K}$ [9]) it shows ferromagnetic (001) planes coupled antiferromagnetically (type-I structure) [9]. We have discussed previously [10] that the calculated lattice constants for ferromagnetic urania agree very well with the experiment while for antiferromagnetic ordering very close values of the lattice constants were found but with unphysical, slightly tetragonal distortion. It is much easier to optimize the geometry of ferromagnetic UN due to a smaller size of the primitive unit cell. Using Quantum Espresso code [5] and generalized gradient approximation PBE (Perdew, Burke, and Ernzerhof) functional [11], we have found the equilibrium lattice constant for ferromagnetic UN to be $5.24 \AA$, which is larger than the experimental value of $4.89 \AA$ [9] since PBE overestimates values of lattice constants.
The used norm conserved potentials provided with Quantum Espresso code (pbe-mt_fhi.UPF) may also lead to a larger lattice constant. 300 Ry cut-off energy and $16 \times 16 \times 16 \mathrm{k}$ points mesh in the Brillouin zone were applied. Our tests show that changing cut-off energy up to $75 \mathrm{Ry}$ and changing grid up to $10 \times 10 \times 10$ have not affected the lattice constant.

Next we performed a band structure calculation for antiferromagnetic and nonmagnetic UN using conventional unit cells (with $5.24 \AA$ lattice constant) and no symmetry. We used 150 Ry cut-off energy and the Monkhorst-Pack [12] 16× $16 \times 16$ grid to calculate the electron band structure suitable as an input to BoltzTraP (BoltzmannTransportProperties) code [6]. This code uses smoothed Fourier interpolation of the electron band structure, which is used to calculate the electron density of states and semiclassic transport coefficients [6]. In Figure 1 the derived electron densities of states (black, solid lines) and their integrals over energy (dashed red lines) are shown for antiferromagnetic (a) and nonmagnetic (b) $\mathrm{UN}$, respectively. These electron densities of state compare well with the one calculated by QE code (Figure 5) presented 


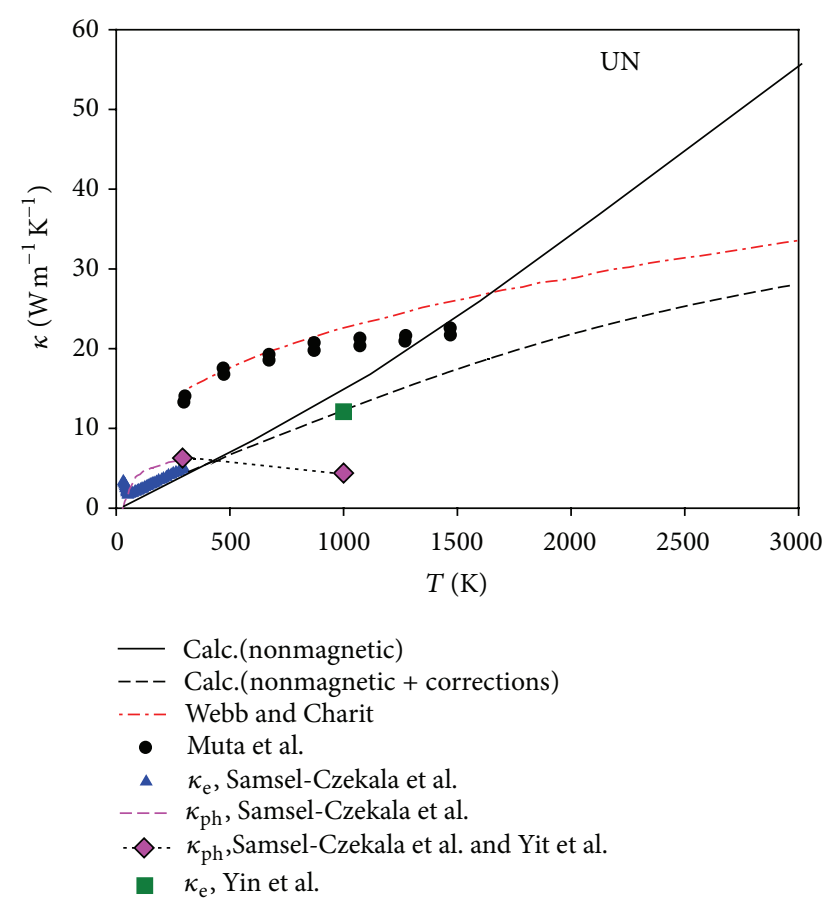

Figure 3: The calculations for thermal conductivity of electronic origin in $\mathrm{UN}$ as a function of temperature are shown for nonmagnetic UN with (short, black, dashed line) and without (black solid line) correction for nonresidual resistivity. The previously estimated phonon contribution to the thermal conductivity at $1000 \mathrm{~K}$ [8] is indicated by a pink diamond and at lower temperatures by a pink dashed line [7] and the electron contribution at $1000 \mathrm{~K}$ [8] is indicated by a green square. The dotted line indicates the phonon contribution evaluated by the linear interpolation between the estimates from $[7,8]$. The electronic contribution, calculated from the experimental resistivity [7], is shown by blue triangles. The measured total thermal conductivity is indicated by the long, red, dashed line [3] and black spheres [16].

in Appendix. In the nonmagnetic calculations one broad peak originating from $5 f$ electrons is visible (b) near the Fermi energy, which splits into two peaks (as discussed in Appendix) for antiferromagnetic ordering (a).

2.2. Uranium Carbide. Next, we performed electronic structure calculations for UC using QE code as described in detail for UN. The same setup of parameters was used. UC has the same crystal structure as UN and we found, similar to $\mathrm{UN}$, the equilibrium lattice constant of $5.26 \AA$ to be larger than the experimental one of $4.958 \AA$ [13]. We also used here norm conserved potentials (pbe-mt_fhi.UPF) provided with Quantum Espresso code. In Figure 2, the electron densities of states of UC used in BolzTrap code are shown and they compare well with the original densities (Figure 6) obtained by $\mathrm{QE}$ code. Similar to UN we see the $5 \mathrm{f}$ electrons of uranium around Fermi energy although the energy of $2 p$ electrons of carbon is about 0.1 Ry higher than the respective energy of $2 p$ electrons of nitrogen in UN (Figure 1).

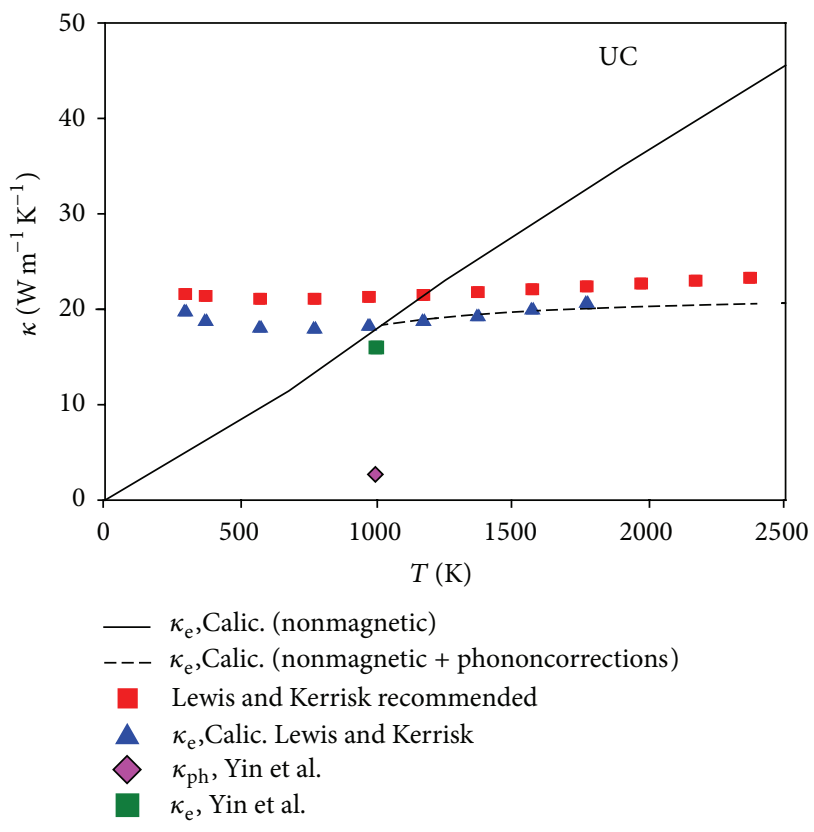

FIGURE 4: The calculations for thermal conductivity of electronic origin in UC as a function of temperature are shown for nonmagnetic UC with (short, black, dashed line) and without (black solid line) correction for nonresidual resistivity. The recommended experimental data for the total thermal conductivity (red squares) and the electronic contribution (blue triangles) to the thermal conductivity [14] are shown. The electron and phonon contributions to the thermal conductivity at $1000 \mathrm{~K}$, evaluated in [8], are indicated by a green square and a pink diamond, respectively.

\section{Thermal Conductivity}

The origin of the high thermal conductivity of UC and UN has been investigated previously. The review [14] of experimental data indicates that the thermal conductivity of UC above $1000^{\circ} \mathrm{C}(1273 \mathrm{~K})$ is $100 \%$ electronic. In [3], the authors state that at temperatures ranging between $300 \mathrm{~K}$ and $2000 \mathrm{~K}$, energy transport of UN is controlled by lattice vibrations (phonons) while at higher temperatures there is significant contribution from conductive electrons. This is in contrast to the other analysis $[7,8]$ indicating a large electronic contribution to transport above $1000 \mathrm{~K}$ and below. Yin et al. have evaluated [8] that the phonon contribution to the thermal conductivity of UN at $1000 \mathrm{~K}$ is only $4.4 \mathrm{~W} \mathrm{~m}^{-1} \mathrm{~K}^{-1}$ and in UC $2.7 \mathrm{~W} \mathrm{~m}^{-1} \mathrm{~K}^{-1}$ versus the electronic contributions of $12.1 \mathrm{~W} \mathrm{~m}^{-1} \mathrm{~K}^{-1}$ and $16 \mathrm{~W} \mathrm{~m}^{-1} \mathrm{~K}^{-1}$, respectively. Researchers have found the electronic contribution to thermal conductivity comparable to phonon transport in UN even below room temperature [7], as we discuss below.

In this work we evaluate only the electronic contribution to the thermal conductivity of UN and UC. The electronic structures of nonmagnetic UN and UC, presented in Section 2, are used in the calculations performed using BoltzTraP code for the temperatures between $50 \mathrm{~K}$ and melting point. 


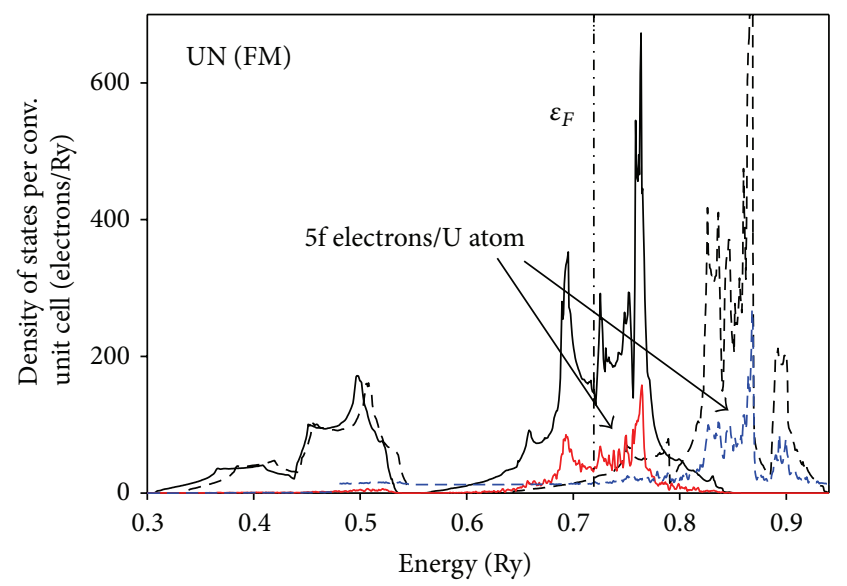

(a)

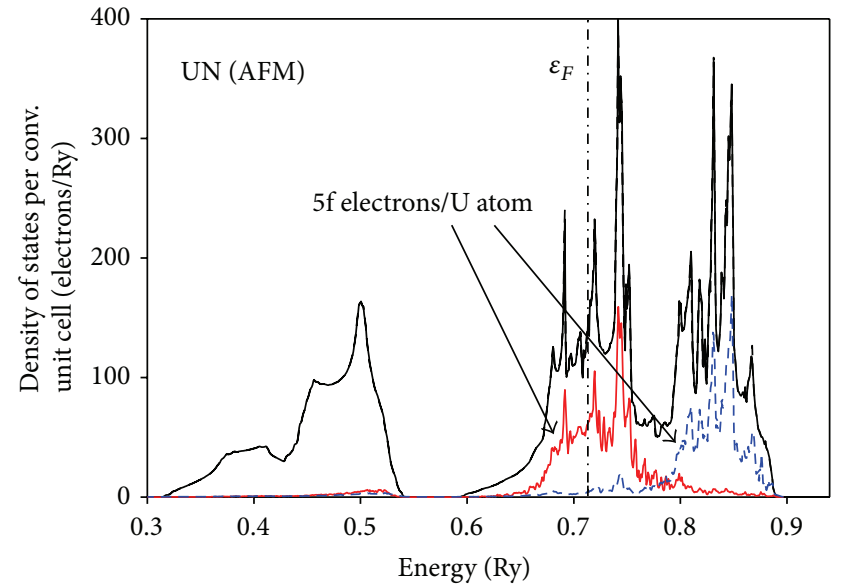

(b)

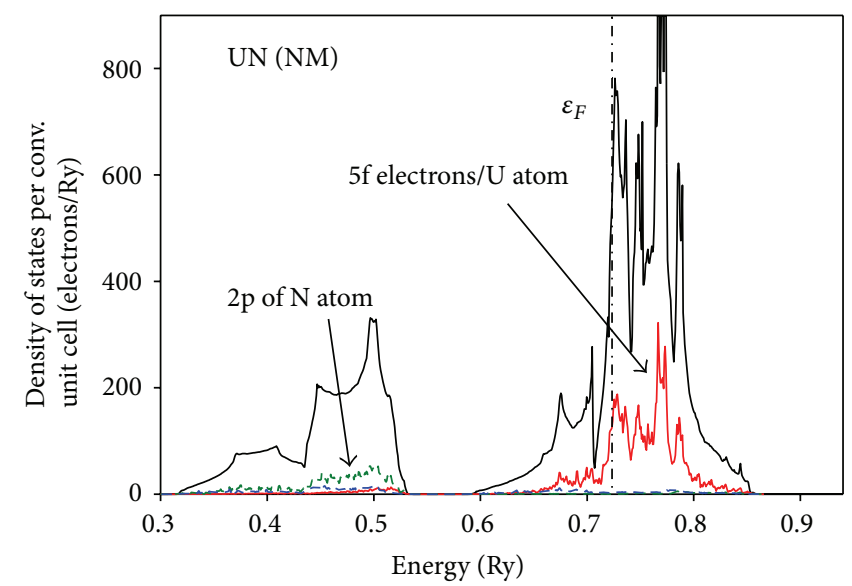

(c)

FIGURE 5: The calculated by QE code the total electron density of states of UN for: (a) ferromagnetic (black, solid line for majority and dashed for minority spins), (b) antiferromagnetic ordering (note identical total majority and minority spin density) and (c) nonmagnetic UN (sum of the density of states of spin up and down electrons plotted by solid line) and conventional unit cell. The densities of states of $5 \mathrm{f}$ electrons of uranium are shown by red solid line and blue dashed line for majority and minority spin on Figures 5(a) and 5(b) and its sum on Figure 5(c).

3.1. Uranium Nitride. In BoltzTraP code it is assumed that the electron relaxation time is isotropic and constant, which is true at lower temperatures where residual resistivity is dominant. We evaluated the relaxation time $(\tau=4.98 \times$ $10^{-15} \mathrm{~s}$ ) using the electronic conductivity, $\sigma / \tau$ equal to 1.18875 $\times 10^{20} \Omega^{-1} \mathrm{~m}^{-1} \mathrm{~s}^{-1}$ calculated by BoltzTraP (for nonmagnetic $\mathrm{UN})$ at $300 \mathrm{~K}$, and the measured resistivity at the same temperature $16.9 \times 10^{-7} \Omega \mathrm{m}$ [7]. However, since the nonresidual resistivity, neglected in BoltztraP, increases linearly with temperature, it becomes important at high temperatures. Interestingly, the previous analysis [8] indicates that in UN approximately $80 \%$ of $\rho(T)$ originates from electronelectron scattering, in contrast to UC where electron-phonon scattering dominates. We evaluated this contribution for UN from the experimental resistivity slope between $300 \mathrm{~K}$ and $1100 \mathrm{~K}[15]$. We found it to be equal to $\Delta_{\rho}=0.4789 \times 10^{-9}$ $(T(K)-300 \mathrm{~K}) \Omega \mathrm{m}$. In Figure 3 we present the calculated electronic contributions to the thermal conductivity $\left(\kappa_{\mathrm{e}}\right)$ of nonmagnetic UN using constant relaxation time, as derived above (black, solid line), and the results with $\Delta_{\rho}$ correction (short, dashed, black line). The electronic contribution to the thermal conductivities was calculated via WiedemannFranz law from the electronic conductivities $(\sigma)$ calculated by BoltzTraP code as follows:

$$
\kappa_{\mathrm{e}}=\frac{\pi}{3}\left(\frac{k_{\mathrm{B}}}{\mathrm{e}}\right)^{2} \sigma T,
$$

where $k_{\mathrm{B}}$ is Boltzmann constant, e is electron charge, and $T$ is the temperature in $\mathrm{K}$.

The previously estimated electronic thermal conductivity at low temperatures [7], shown by blue triangles in Figure 3, is in very good agreement with the values calculated here for nonmagnetic UN and is above Néel temperature $(51 \mathrm{~K})$. The phonons' contribution to the thermal conductivity (indicated by dashed and dotted pink lines) decreases with temperature [7] and therefore around $1500 \mathrm{~K}$ of our estimate of the electronic contribution to the thermal conductivity of nonmagnetic UN (black solid line) agrees well with the measured 


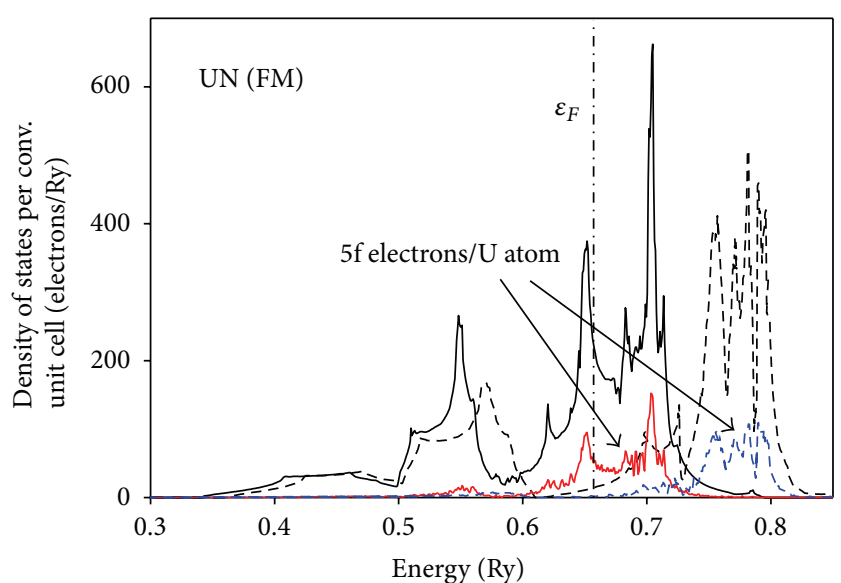

(a)

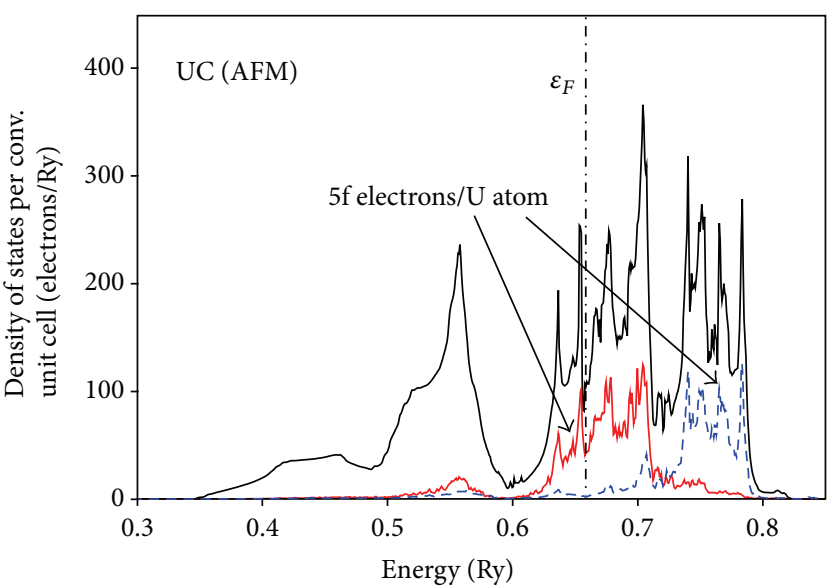

(b)

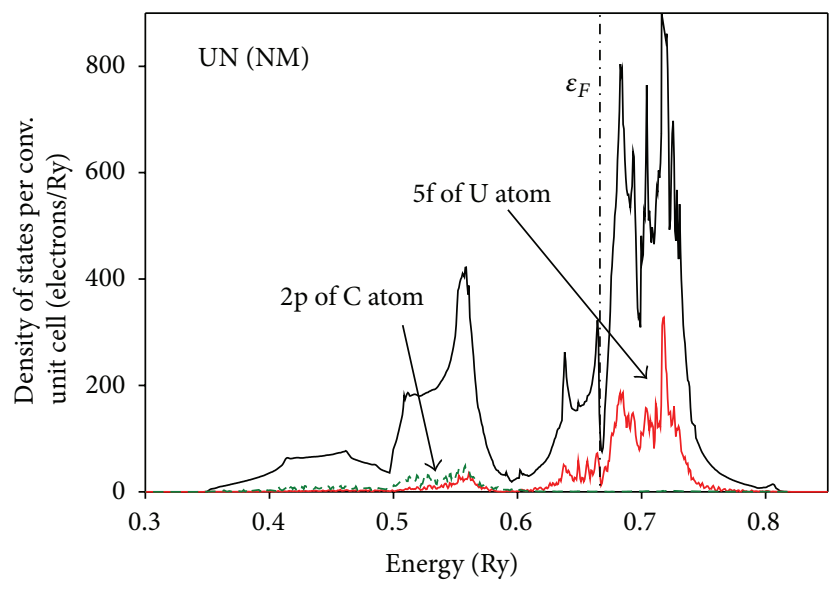

(c)

FIGURE 6: The calculated by QE code the total electron density of states of UC for: (a) ferromagnetic (black, solid line for majority and dashed for minority spins), (b) antiferromagnetic ordering (note identical total majority and minority spin density) and (c) nonmagnetic UC (sum of the density of states of spin up and down electrons plotted by solid line) and conventional unit cell. The densities of states of $5 \mathrm{f}$ electrons of uranium are shown by red solid line and blue dashed line for majority and minority spin on Figures 6(a) and 6(b) and its sum on Figure 6(c).

thermal conductivity (long, red, dashed line [3], and black spheres [16]). However the overprediction of the thermal conductivity at higher temperature makes questionable the assumption used in BoltzTraP that the relaxation time is constant for the whole range of temperatures and therefore we also include the thermal conductivity calculated using the correction for the nonresidual resistivity (short, dashed black lines), which at $1000 \mathrm{~K}$ is in very good agreement with the value (indicated by green square) reported in [8].

There is, however, disagreement between experimental data for resistivity (e.g., at $300 \mathrm{~K}$ resistivity: $16.9 \times 10^{-7} \Omega \mathrm{m}$ [7] versus $14.8 \times 10^{-7} \Omega \mathrm{m}$ [15]) and thermal conductivity, as shown in Figure 3 (e.g., around $1500 \mathrm{~K}$, conductivity: $22 \mathrm{~W} \mathrm{~m}^{-1} \mathrm{~K}^{-1}[16]$ versus $26 \mathrm{~W} \mathrm{~m}^{-1} \mathrm{~K}^{-1}$ [3]). The band structure of uranium $5 \mathrm{f}$ electrons is also not very well represented within DFT, as discussed before [8]. However, our calculations reproduce very well the trend in the thermal conductivity of UN to increase with temperature.
3.2. Uranium Carbide. The relaxation time of UC ( $\tau=5.79$ $\times 10^{-15} \mathrm{~s}$ ) was evaluated using the experimental value of resistivity $13.4 \times 10^{-7} \Omega \mathrm{m}[14]$ at $1000 \mathrm{~K}$ and the electronic conductivity, $\sigma / \tau$ equal to $1.2886 \times 10^{20} \Omega^{-1} \mathrm{~m}^{-1} \mathrm{~s}^{-1}$, calculated by BoltzTraP (for nonmagnetic UC) at the same temperature. As with UN, we evaluated the nonresidual contribution to the resistivity of UC from the experimental resistivity slope between $298 \mathrm{~K}$ and $1773 \mathrm{~K}$ [14]. We used this correction above $1000 \mathrm{~K}$ and found the slope two times steeper than in UN and the correction equal to $\Delta_{\rho}=0.1079 \times$ $10^{-8}(T(\mathrm{~K})-1000 \mathrm{~K}) \Omega \mathrm{m}$.

In Figure 4, we show by solid line the electronic contribution to the thermal conductivities calculated via WiedemannFranz law (1) from the electronic conductivities as a function of temperature. The electronic conductivities were derived using BoltzTraP. The relaxation time evaluated above was used. The recommended experimental data for both the total thermal conductivity (red squares) and the electronic contribution (blue triangles) to the thermal conductivity 
[14] show very little variation with temperature, which is in disagreement with the calculation.

However, when the correction for electron-phonon interaction is taken into account (broken, black line) above $1000 \mathrm{~K}$, a very good agreement with experiment is obtained. The previously [8] calculated electron and phonon contribution to the thermal conductivity of UC at $1000 \mathrm{~K}$ is indicated by green square and pink diamond, respectively, and predict slightly lower the total thermal conductivity of UC $\left(18.7 \mathrm{Wm}^{-1} \mathrm{~K}^{-1}\right)$ than experiment $\left(21.3 \mathrm{Wm}^{-1} \mathrm{~K}^{-1}\right)$ [14]. The estimated in [8] electron contribution to the thermal conductivity: $16 \mathrm{Wm}^{-1} \mathrm{~K}^{-1}$ is also lower than calculated here: $\kappa_{\mathrm{e}}=18.2 \mathrm{Wm}^{-1} \mathrm{~K}^{-1}$. Our analysis confirms that in evaluation of the thermal conductivity of UC, the effect of phonons needs to be taken into account.

\section{Summary}

We demonstrate that, by combining first-principles quantum-mechanical calculations with classical correlations, it is possible to predict the thermal conductivity of UN and UC in qualitative agreement with experiment.

The electronic structure was calculated using Quantum Espresso code. BoltzTrap code was used to evaluate electronic conductivity for nonmagnetic UN and UC, which was used to estimate the electronic contribution to the thermal conductivities via Wiedemann-Franz law. The relaxation time was derived using the experimental values of resistivity.

The metallic fuels like UN and UC show high electronic contribution to thermal conductivity, which increases with temperature and therefore makes them more suitable as fuels for some new generation reactors, as, for example, SCWR and fast reactors.

\section{Appendix}

In this section the electronic densities of states as calculated by QE code [5] are presented. Similar to our earlier work on uranium oxides [10, 17], spin polarized and nonspin polarized calculations were performed without spin orbital effect included. As described in Section 2 the ferromagnetic ordering on $\mathrm{U}$ lattice was assumed to evaluate the equilibrium lattice constants of UN and UC at $0 \mathrm{~K}$, and therefore primitive unit cell could be used (with only two atoms) during geometry optimization calculations. In Figures 5 and 6, the calculated densities of states are shown using the respective equilibrium lattice constants: $5.24 \AA$ (UN) and $5.26 \AA$ (UC). The presented here densities of states were calculated using PBE functional [11]. We used 150 Ry cut-off energy and the dense Monkhorst-Pack [12] $16 \times 16 \times 16$ grid for higher accuracy. Figures 5(a) and 5(b) and Figures 6(a) and 6(b) represent spin polarized calculation for ferromagnetic and antiferromagnetic ordering on $U$ lattice, while Figures 5(c) and $6(\mathrm{c})$ are for nonmagnetic UN and UC, respectively.

The presented here on Figures 5 and 6 electron densities of states as obtained by QE code agree very well with that derived by BoltzTrap code and shown on Figures 1 and 2 . In Figures 5 and 6 the calculations for ferromagnetic ordering
TABLE 1: Magnetic moment on U in U compounds.

\begin{tabular}{lccc}
\hline Compound & \multicolumn{3}{c}{$M_{\mathrm{U}}\left[\mu_{\mathrm{B}}\right]$} \\
& Löwdin [18] & Mulliken [19] & Hirshfeld [20] \\
\hline UN (AFM) & 2.29 & 2.88 & 2.64 \\
UN (FM) & 2.74 & 3.02 & 2.86 \\
UC (AFM) & 1.78 & 2.74 & 2.40 \\
UC (FM) & 2.04 & 2.70 & 2.50 \\
$\mathrm{UO}_{2}$ (FM) [10,17] & - & 2.14 & 2.02 \\
$\mathrm{UO}_{2}$ (AFM) $[10,17]$ & - & 2.10 & 1.96 \\
\hline
\end{tabular}

are shown additionally. The minority spin density of states is represented by broken line, while majority electron spin density is shown by solid line.

The density of states per one $U$ atom of spin-up and -down $5 f$ electrons is indicated by red and blue lines and it can be easily confirmed that the broad peak around the Fermi energy of the total density of states (plotted for the conventional unit cell and containing four $U$ atoms) originates from its majority spin electron density. The same is true for antiferromagnetic ordering (Figures 5(b) and 6(b)).

As discussed before for $\mathrm{UO}_{2}$ [10] there is a big similarity between antiferromagnetic and ferromagnetic densities of states of uranium compounds.

In Figures 5(c) and 6(c) the electron densities are shown for nonmagnetic UC and UN. It can be confirmed that there is only one broad peak near the Fermi energy that originates from 5 f electrons of $U$ atoms. The peak in the density of states at lower energy originates predominantly from $2 \mathrm{p}$ electrons of $\mathrm{N}$ and $\mathrm{C}$ atoms as indicated. However there is also smaller contribution from $5 \mathrm{f}$ and $6 \mathrm{~d}$ electrons of $\mathrm{U}$, which is larger for UC due to a stronger hybridization with $2 p$ electrons of carbon.

Although only the non-spin-polarised calculations are used for thermal conductivity calculations (see Section 3) for completeness, we are presenting here also the details on the polarization calculated using $\mathrm{QE}$ code. Similar to our earlier work on $\mathrm{UO}_{2}$ [10], polarization on uranium atoms in UC and UN is evaluated in Table 1 and compared for ferromagnetic (FM) and antiferromagnetic ordering (AFM). The calculations for $\mathrm{UC}$ and $\mathrm{UN}$ were done using $\mathrm{PBE}$ functional [11] and QE code [5]. Löwdin [18] method was used to extract presented in Table 1 magnetic moments. Additionally for comparison we present the predicted local moments on uranium by CASTEP code using local spin density with Hubbard $\mathrm{U}$ correction [10] $\left(3.5 \mathrm{eV}\right.$ for $\mathrm{UO}_{2}$ $[10,17]$ and $4.5 \mathrm{eV}$ for UX compounds) and Mulliken [19] and Hirshfeld [20] analysis.

As discussed in [7] the distance U-U in UX compounds is large and therefore the calculated here local magnetic moments should be compared to the evaluated experimentally effective moment from susceptibility equal to $\sim 2.66 \mu_{B}$. This value compares well with the calculated values presented in Table 1 for UN and UC for both ferromagnetic (FM) and antiferromagnetic ordering (AFM). Both QE and CASTEP predict lower moment in UC that can be explained by 
discussed above stronger hybridization between $2 p$ electrons of carbon and $5 \mathrm{f}$ electrons of uranium.

\section{Conflict of Interests}

The authors declare that there is no conflict of interests regarding the publication of this paper.

\section{Acknowledgments}

The authors acknowledge access to high performance supercomputers at CLUMEQ, Westgrid, and Plato and collaboration with Ki-Seob Sim and I. L. Pioro on advanced nuclear fuels.

\section{References}

[1] I. L. Pioro, M. Khan, V. Hopps et al., "SCW pressure channel nuclear reactor. Some design features," Journal of Power and Energy Systems, vol. 2, pp. 874-888, 2008.

[2] P. L. Kirillov, Ed., Thermophysical Properties of Materials for Nuclear Engineering, Tutorial for Students of Specialty Nuclear Power Plants, Obninsk, Russia, 2nd revised and augmented edition, 2006.

[3] J. A. Webb and I. Charit, "Analytical determination of thermal conductivity of $\mathrm{W}-\mathrm{UO}_{2}$ and W-UN CERMET nuclear fuels," Journal of Nuclear Materials, vol. 427, pp. 87-94, 2012.

[4] B. M. Ma, Nuclear Reactor Materials and Applications, Van Nostrand Reinhold Company, Technology \& Engineering, 1983.

[5] P. Giannozzi, S. Baroni, N. Bonini et al., "QUANTUM ESPRESSO: a modular and open-source software project for quantum simulations of materials," Journal of Physics Condensed Matter, vol. 21, no. 39, Article ID 395502, 2009.

[6] G. K. H. Madsen and D. J. Singh, "BoltzTraP. A code for calculating band-structure dependent quantities," Computer Physics Communications, vol. 175, no. 1, pp. 67-71, 2006.

[7] M. Samsel-Czekala, E. Talik, P. Du Plessis, R. Troć, H. Misiorek, and C. Sułkowski, "Electronic structure and magnetic and transport properties of single-crystalline UN," Physical Review B, vol. 76, Article ID 144426, 16 pages, 2007.

[8] Q. Yin, A. Kutepov, K. Haule, G. Kotliar, S. Y. Savrasov, and W. E. Pickett, "Electronic correlation and transport properties of nuclear fuel materials," Physical Review B, vol. 84, no. 19, Article ID 195111, 2011.

[9] H. W. Knott, G. H. Lander, M. H. Mueller, and O. Vogt, "Search for lattice distortions in UN, UAs, and USb at low temperatures," Physical Review B, vol. 21, no. 9, pp. 4159-4165, 1980.

[10] B. Szpunar and J. A. Szpunar, "Application of density functional theory in assessing properties of thoria and recycled fuels," Journal of Nuclear Materials, vol. 439, no. 1-3, pp. 243-250, 2013.

[11] J. P. Perdew, K. Burke, and M. Ernzerhof, "Generalized gradient approximation made simple," Physical Review Letters, vol. 77, no. 18, pp. 3865-3868, 1996.

[12] H. J. Monkhorst and J. D. Pack, "Special points for Brillouinzone integrations," Physical Review B: Solid State, vol. 13, no. 12, pp. 5188-5192, 1976.

[13] M. Hisayuki, S. Ken-ichi, I. Mitsuo, A. Hiromi, and K. Tomoo, "Electrical resistivity and lattice parameter of uranium monocarbide," Journal of Nuclear Materials, vol. 57, no. 1, pp. 93-97, 1975.
[14] H. D. Lewis and J. F. Kerrisk, "Electrical and thermal transport properties of uranium and plutonium carbides: a review of the literature," Tech. Rep. LA-6096, Los Alamos National Laboratory, 1976.

[15] T. Ohmichi, T. Kikuchi, and S. Nasu, "Electrical resistivity and thermoelectric power of $\mathrm{UC}^{1-\mathrm{x}} \mathrm{N}_{\mathrm{x}}$," Journal of Nuclear Science and Technology, vol. 9, pp. 77-85, 1972.

[16] H. Muta, K. Kurosaki, M. Uno, and S. Yamanaka, “Thermal and mechanical properties of uranium nitride prepared by SPS technique," Journal of Materials Science, vol. 43, no. 19, pp. 64296434, 2008.

[17] B. Szpunar, J. A. Szpunar, V. Milman, and A. Goldberg, "Implication of volume changes in uranium oxides: a density functional study," Solid State Sciences, vol. 24, pp. 44-53, 2013.

[18] P. Löwdin, "On the non-orthogonality problem connected with the use of atomic wave functions in the theory of molecules and crystals," The Journal of Chemical Physics, vol. 18, no. 3, pp. 365375, 1950.

[19] R. S. Mulliken, "Electronic population analysis on LCAO-MO molecular wave functions," The Journal of Chemical Physics, vol. 23, no. 10, pp. 1833-1840, 1955.

[20] F. L. Hirshfeld, "Bonded-atom fragments for describing molecular charge densities," Theoretica Chimica Acta, vol. 44, no. 2, pp. 129-138, 1977. 


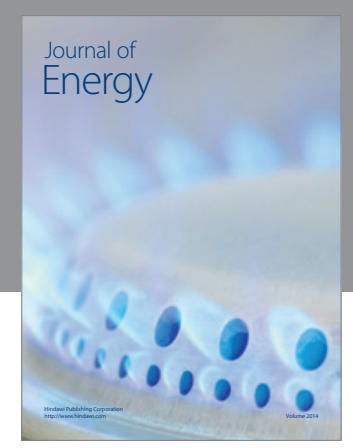

Journal of

Industrial Engineering
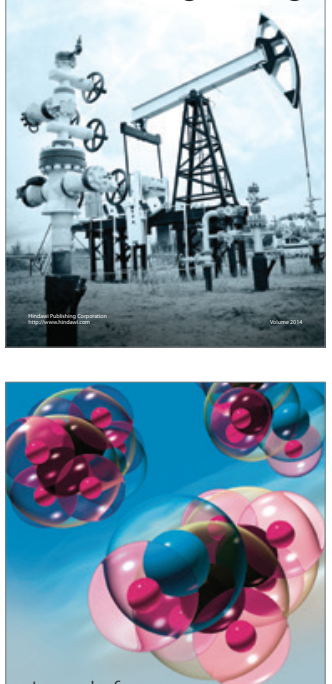

Fuels
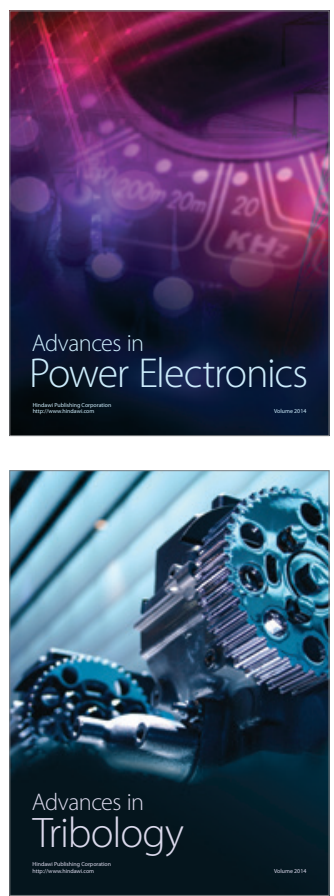

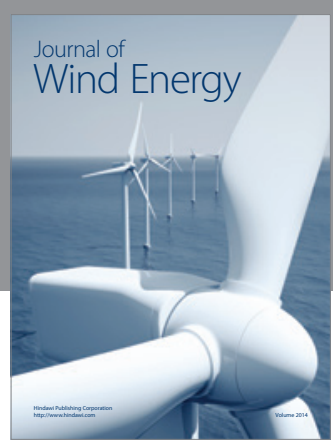

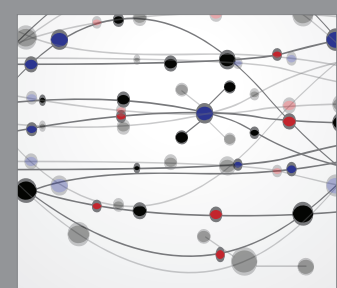

The Scientific World Journal

Submit your manuscripts at http://www.hindawi.com

Journal of

Structures
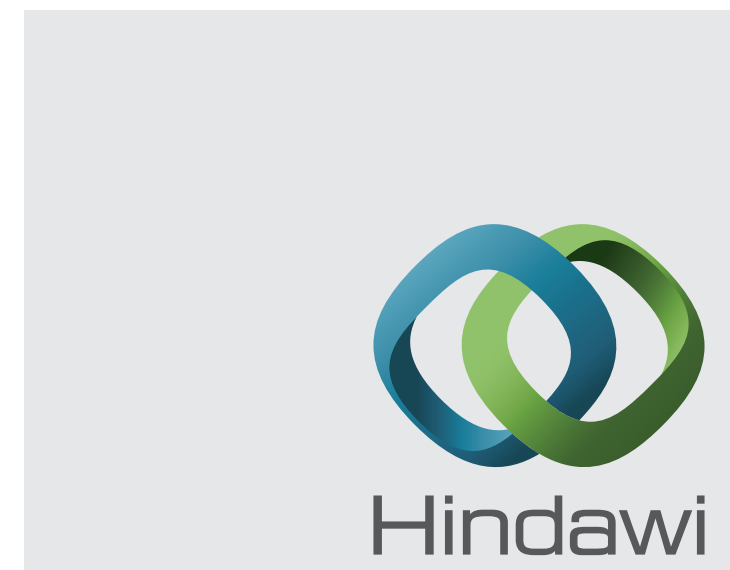

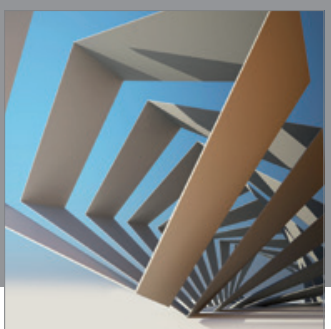

Rotating

Machinery
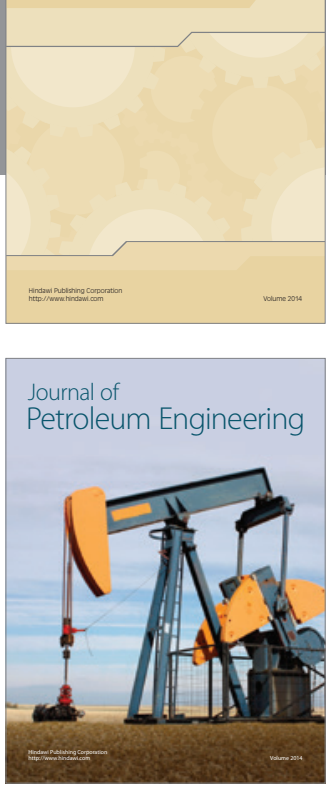

Journal of

Solar Energy
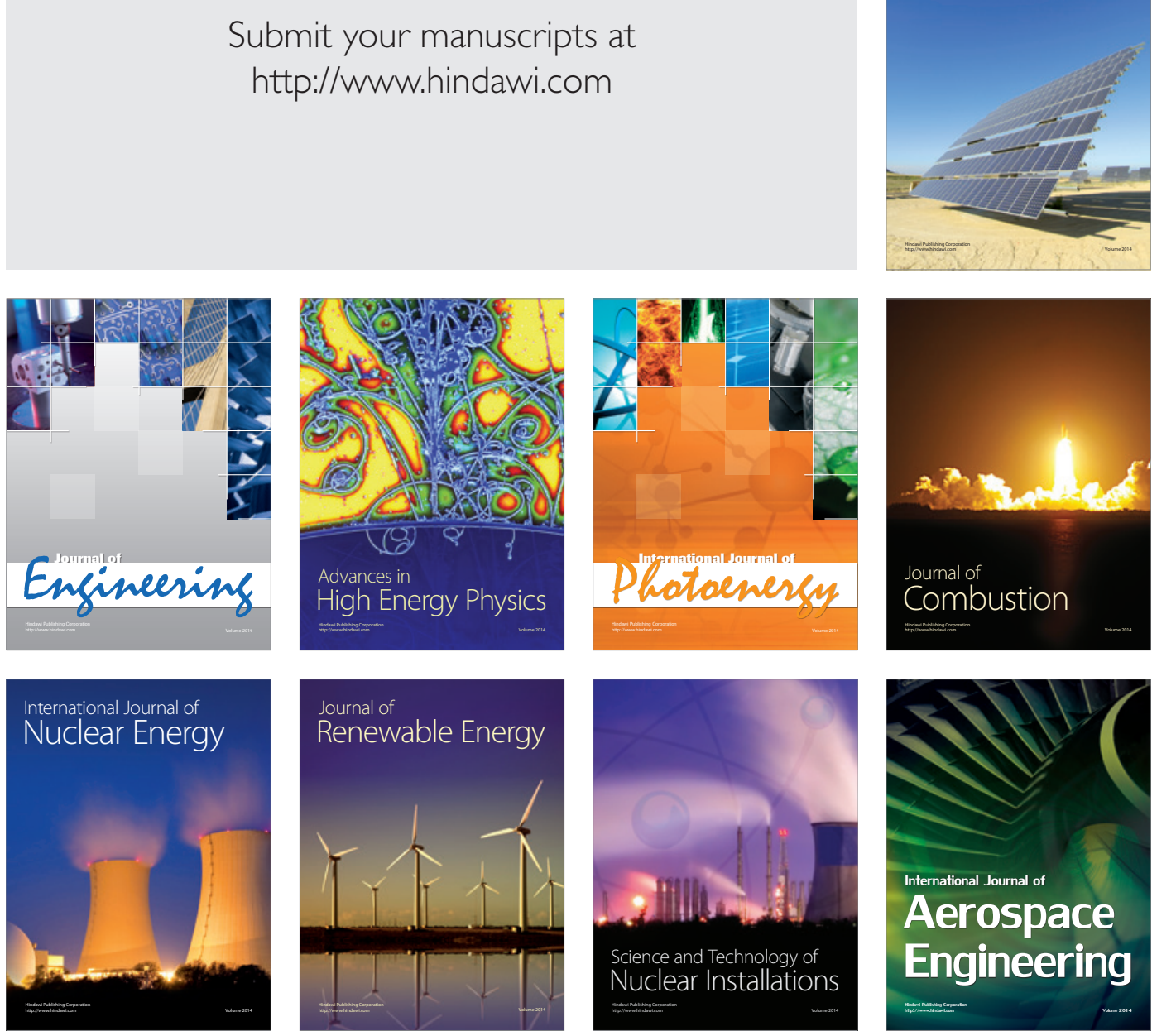